\title{
Strongly Intensive Measures for Transverse Momentum and Particle Number Fluctuations
}

\author{
Mark I. Gorenstein ${ }^{1,2}$ and Katarzyna Grebieszkow ${ }^{3}$ \\ ${ }^{1}$ Bogolyubov Institute for Theoretical Physics, Kiev, Ukraine \\ ${ }^{2}$ Frankfurt Institute for Advanced Studies, Frankfurt, Germany \\ ${ }^{3}$ Faculty of Physics, Warsaw University of Technology, Warsaw, Poland
}

\begin{abstract}
Strongly intensive measures $\Delta\left[P_{T}, N\right]$ and $\Sigma\left[P_{T}, N\right]$ are used to study the event-by-event fluctuations of the transverse momentum $P_{T}$ and particle multiplicity $N$ in nucleus-nucleus collisions. A special normalization for these fluctuation measures ensures that they are dimensionless and yields a common scale required for a quantitative comparison of fluctuations. In this paper basic properties of the $\Delta\left[P_{T}, N\right]$ and $\Sigma\left[P_{T}, N\right]$ measures are tested within different phenomenological models using the Monte Carlo simulations (the so-called fast generators) and analytical solutions. The obtained results are helpful to elucidate the properties of the $\Delta\left[P_{T}, N\right]$ and $\Sigma\left[P_{T}, N\right]$ measures.

PACS numbers: 12.40.-y, 12.40.Ee
\end{abstract}

Keywords: 


\section{INTRODUCTION}

The main motivation for the experiments studies on relativistic nucleus-nucleus $(\mathrm{A}+\mathrm{A})$ collisions is to create and study strongly interacting matter. Experimental and theoretical investigations of event-by-event (e-by-e) fluctuations in $\mathrm{A}+\mathrm{A}$ collisions produce new information about its properties. E-by-e fluctuations can be also an important tool for localizing the phase boundary and the critical point of the QCD matter. In particular, significant increase of transverse momentum and multiplicity fluctuations are expected in a vicinity of the critical point [1]. One can probe different regions of the phase diagram by varying the collision energy and the size of colliding nuclei [2]. A possibility to observe signatures of the critical point inspired the energy and system size scan program of the NA61/SHINE Collaboration at the CERN Super Proton Synchrotron (SPS) [3] and the low energy scan program of the STAR and PHENIX Collaborations at the Brookhaven National Laboratory's Relativistic Heavy Ion Collider (BNL RHIC) [4]. In these studies one measures and then compares the e-by-e fluctuations in collisions of different nuclei at different collision energies. The average sizes of created physical systems and their e-by-e fluctuations are expected to be rather different [5]. This strongly affects the observed hadron fluctuations, and consequently the measured quantities do not describe local physical properties of the system but rather reflect the system size fluctuations. For instance, $\mathrm{A}+\mathrm{A}$ collisions with different centralities may produce a system with approximately the same local properties (e.g., the same temperature and baryonic chemical potential) but with the volume changing significantly from interaction to interaction. Note that in high energy collisions the average volume of created matter and its variations from collision to collision are usually out of experimental control, i.e. the volume variations are difficult or even impossible to measure. Therefore, a suitable choice of statistical tools for the study of e-by-e fluctuations is really important.

In statistical mechanics, an extensive quantity is proportional to the system volume $V$, whereas an intensive one has fixed finite value in the thermodynamical limit $V \rightarrow \infty$. Intensive quantities are used to describe local properties of a physical system. In particular, the equation of state of the matter is usually formulated in terms of intensive physical quantities, e.g., the pressure is considered as a function of temperature and chemical potentials. 
The strongly intensive quantities have been introduced in Ref. [6]. Within the grand canonical ensemble formulation of statistical mechanics they are independent of the average volume and volume fluctuations. Similar properties take place in the model of independent sources: the strongly intensive measures of fluctuations are independent of the average number of sources and of fluctuations of the number of sources. The strongly intensive measures $\Delta[A, B]$ and $\Sigma[A, B]$ are suggested for studies of e-by-e fluctuations of hadron production in heavy ion collisions at high energies. They are defined using two arbitrary extensive quantities $A$ and $B$. In the present paper we consider a pair of extensive variables: the transverse momentum $A=P_{T}=p_{T}^{(1)}+\ldots p_{T}^{(N)}$, where $p_{T}^{(i)}$ is the absolute value of the $i^{\text {th }}$ particle transverse momentum, and the number of particles $B=N$. The measures $\Delta\left[P_{T}, N\right]$ and $\Sigma\left[P_{T}, N\right]$ were studied recently within the ultra-relativistic quantum molecular dynamics (UrQMD) simulations in Ref. [7]. The measures $\Delta[A, B]$ and $\Sigma[A, B]$ in the case of two hadron multiplicities $A$ and $B$ were considered within the hadron-string dynamics (HSD) transport model in Ref. [8]. To simplify notations we sometimes use $X=P_{T}$ and $x_{i}=p_{T}^{(i)}$. Note that our consideration is valid also for other motional variables $X$, e.g., the system energy $X=E=\epsilon_{1}+\cdots+\epsilon_{N}$. The strongly intensive measure $\Delta[X, N]$ and $\Sigma[X, N]$ are defined as [6]:

$$
\begin{aligned}
& \Delta[X, N]=\frac{1}{C_{\Delta}}[\langle N\rangle \omega[X]-\langle X\rangle \omega[N]], \\
& \Sigma[X, N]=\frac{1}{C_{\Sigma}}[\langle N\rangle \omega[X]+\langle X\rangle \omega[N]-2(\langle X N\rangle-\langle X\rangle\langle N\rangle)],
\end{aligned}
$$

where

$$
\omega[X]=\frac{\left\langle X^{2}\right\rangle-\langle X\rangle^{2}}{\langle X\rangle}, \quad \omega[N]=\frac{\left\langle N^{2}\right\rangle-\langle N\rangle^{2}}{\langle N\rangle}
$$

are the scaled variances for $X$ and $N$ fluctuations, and $C_{\Delta}$ and $C_{\Sigma}$ are normalization factors. The notation $\langle\ldots\rangle$ represents the e-by-e averaging.

The first strongly intensive measure of fluctuations, the so-called $\Phi$ measure, was introduced a long time ago in Ref. [9]. There were many attempts to use the $\Phi$ measure in the data analysis [10 16] and in theoretical models [17 32]. In general, $\Phi$ is a dimensional quantity and it does not assume a characteristic scale for a quantitative analysis of e-by-e fluctuations for different observables. Note that the latter properties were clearly disturbing. 
In the recent paper [33] special normalization has been proposed for the $\Delta$ and $\Sigma$ fluctuation measures. It is used in the present study and ensures that measures (1) and (2) are dimensionless and yields a common scale required for a quantitative comparison of the e-by-e fluctuations. This normalization has been already used for the $\Delta\left[P_{T}, N\right]$ and $\Sigma\left[P_{T}, N\right]$ measures using the transport model of A+A collisions in Ref. [33] and for the ideal quantum gases within the grand canonical ensemble formulation [34]. Note that the NA61 Collaboration has already started to use the strongly intensive measures to study e-by-e fluctuations in A+A collisions [35].

In the present paper several phenomenological models of hadron production are suggested and studied using the Monte Carlo (MC) simulations (the so-called fast generators). Analytical solutions for the proposed models are also presented and analyzed. These studies are helpful to elucidate properties of the $\Delta\left[P_{T}, N\right]$ and $\Sigma\left[P_{T}, N\right]$ measures. A search for possible signals for the phase transition and critical point in $\mathrm{A}+\mathrm{A}$ collisions is outside of the scope of our paper. To achieve this goal one first needs to formulate suitable dynamical models for these phenomena.

The paper is organized as follows. In Sec. II we introduce two reference models. The first model is the independent particle model within which we calculate the normalization factors $C_{\Delta}$ and $C_{\Sigma}$ for $\Delta[X, N]$ and $\Sigma[X, N]$ quantities. The second model is the model of independent sources which is often used to analyze the data on nucleus-nucleus collisions. Section III presents examples of the MC simulations. Some of these examples correspond to different versions of the model of independent sources. Analytical solutions are also presented and analyzed. In Sec. IV the MC simulations and analytical consideration are used for the models where single particle momentum spectra are dependent on the number of the produced particles. In Sec. $\mathrm{V}$ results of statistical and transport models are presented. Using the UrQMD simulations we study effects of the centrality selection and limited detector acceptance and efficiency in $\mathrm{A}+\mathrm{A}$ collisions. A summary in Sec. VI closes the article.

\section{REFERENCE MODELS}

In this section two simple models of particle production are presented. The first one is the independent particle model (IPM) which is used as a reference model to fix the normalization of the strongly intensive measures $\Delta$ and $\Sigma$. Namely, properly normalized strongly intensive 
quantities assume the value one for the fluctuations given by the IPM. The second model is the model of independent sources. In this model, the values of $\Delta$ and $\Sigma$ for the system of sources are equal to their values for a single source.

\section{A. Independent Particle Model}

In Ref. [33] a special normalization for the strongly intensive measures $\Delta[A, B]$ and $\Sigma[A, B]$ has been proposed. In this subsection we present its derivation when $A$ is an extensive variable $A=X$ presented as a sum of single particle terms

$$
X=x_{1}+x_{2}+\ldots+x_{N}
$$

(e.g., the system energy $E$ or transverse momentum $P_{T}$ ) and $B=N$ is the number of particles. Inter-particle correlations are absent in the IPM, i.e. the probability of any multi-particle state is a product of probability distributions $F\left(x_{j}\right)$ of single-particle variables $x_{j}$, and these probability distributions are the same for all $j=1, \ldots, N$ and independent of the number of particles $N$ :

$$
F_{N}\left(x_{1}, x_{2}, \ldots, x_{N}\right)=\mathcal{P}(N) \times F\left(x_{1}\right) F\left(x_{2}\right) \times \cdots \times F\left(x_{N}\right)
$$

where $\mathcal{P}(N)$ is an arbitrary multiplicity distribution of particles. The functions entering Eq. (5) satisfy the normalization conditions:

$$
\sum_{N} \mathcal{P}(N)=1, \quad \int d x F(x)=1 .
$$

The averaging procedure for $k^{\text {th }}$ moments of any multiparticle observable $A$ reads:

$$
\left\langle A^{k}\right\rangle=\sum_{N} \mathcal{P}(N) \int d x_{1} d x_{2} \ldots d x_{N} F\left(x_{1}\right) F\left(x_{2}\right) \times \cdots \times F\left(x_{N}\right)\left[A\left(x_{1}, x_{2}, \ldots, x_{N}\right)\right]^{k} .
$$

For the first and second moments of $X$ and $N$ one obtains:

$$
\langle X\rangle=\bar{x} \cdot\langle N\rangle, \quad\left\langle X^{2}\right\rangle=\overline{x^{2}} \cdot\langle N\rangle+\bar{x}^{2} \cdot\left[\left\langle N^{2}\right\rangle-\langle N\rangle\right], \quad\langle X N\rangle=\bar{x} \cdot\left\langle N^{2}\right\rangle
$$

where

$$
\left\langle N^{k}\right\rangle=\sum_{N} \mathcal{P}(N) N^{k}, \quad \overline{x^{k}}=\int d x F(x) x^{k}
$$


Note that the overline denotes averaging over single particle inclusive distribution, whereas $\langle\ldots\rangle$ represents event averaging over multiparticle states of the system, e.g., e-by-e averaging over hadrons detected in $\mathrm{A}+\mathrm{A}$ collisions.

Using Eq. (8), one finds

$$
\begin{aligned}
& \omega[X] \equiv \frac{\left\langle X^{2}\right\rangle-\left\langle X^{2}\right\rangle}{\langle X\rangle}=\frac{\overline{x^{2}}-\bar{x}^{2}}{\bar{x}}+\bar{x} \cdot \frac{\left\langle N^{2}\right\rangle-\langle N\rangle^{2}}{\langle N\rangle} \equiv \omega[x]+\bar{x} \cdot \omega[N], \\
& \langle X N\rangle-\langle X\rangle\langle N\rangle=\bar{x} \cdot\left[\left\langle N^{2}\right\rangle-\langle N\rangle^{2}\right] \equiv \bar{x} \cdot\langle N\rangle \omega[N]
\end{aligned}
$$

and finally,

$$
\begin{aligned}
& \Delta[X, N]=\frac{1}{C_{\Delta}}[\langle N\rangle \omega[X]-\langle X\rangle \omega[N]]=\frac{\omega[x] \cdot\langle N\rangle}{C_{\Delta}}, \\
& \Sigma[X, N]=\frac{1}{C_{\Sigma}}[\langle N\rangle \omega[X]+\langle X\rangle \omega[N]-2(\langle X N\rangle-\langle X\rangle\langle N\rangle)]=\frac{\omega[x] \cdot\langle N\rangle}{C_{\Sigma}} .
\end{aligned}
$$

The requirement that

$$
\Delta[X, N]=\Sigma[X, N]=1
$$

for the IPM leads thus to the normalization factors

$$
C_{\Delta}=C_{\Sigma}=\omega[x] \cdot\langle N\rangle, \quad \omega[x] \equiv \frac{\overline{x^{2}}-\bar{x}^{2}}{\bar{x}} .
$$

The normalization factors (15) are suggested to be used both in theoretical models and for the data analysis (see Ref. [33] for further details of the normalization procedure).

According to the current classification the $\Phi$ measure [9] belongs to the $\Sigma$ family [6]. It can be calculated as

$$
\Phi_{X}=[\bar{x} \omega[x]]^{1 / 2}[\sqrt{\Sigma[X, N]}-1]
$$

The representation of $X$ with Eq. (4) as the sum of single particle variables $x_{i}$ is an evident feature of the IPM. Thus, one needs such a representation to calculate the normalization factors $C_{\Delta}$ and $C_{\Sigma}$. Such a representation of the extensive motional variable is, however, not necessarily needed for the e-by-e measurements. For example, the system energy $E$ (or transverse momentum $P_{T}$ ) can be measured by a calorimeter without determining individual single particle contributions. 
It was proven [33] that the IPM relation (14) is valid also in two models. The first model is statistical mechanics for the Boltzmann ideal gas within the grand canonical ensemble. The second model is the mixed event procedure which creates a sample of artificial events, where each particle is taken from different physical events. These model constructions play an important role as reference model. The deviations of real data from the IPM results (14) can be used to clarify the physical properties of the system. It resembles the situation with particle number distributions. One prefers to use the Poisson distribution $P(N)=\exp (-\bar{N}) \bar{N}^{N} / N$ ! with $\omega[N]=1$ as a reference model. Another reference value $\omega[N]=0$ corresponds to $N=$ const, where the $N$-fluctuations are absent. The fluctuations for any particle number distribution $\mathcal{P}(N)$ is then clarified by the comparison of the calculated (or measured) scaled variance $\omega[N]$ with its reference value of $\omega[N]=1$. The relation $\omega[N]>1$ (or $\omega[N] \gg 1$ ) corresponds to "large" (or "very large") fluctuations of $N$, and $\omega[N]<1$ (or $\omega[N] \ll 1$ ) to "small" (or "very small") fluctuations.

\section{B. Model of Independent Sources}

In this subsection we consider a model of independent sources (MIS) for multi-particle production. In this model the number of sources, $N_{S}$, changes from event to event. The sources are statistically identical and independent of each other. A famous example of the MIS is the wounded nucleon model [36] for A+A collisions . Two fluctuating extensive quantities $X$ and $N$ can be expressed as

$$
X=X_{1}+X_{2}+\ldots+X_{N_{S}}, \quad N=n_{1}+n_{2}+\ldots+n_{N_{S}},
$$

where $n_{j}$ denotes the number of particles emitted from the $j^{\text {th }}$ source $\left(j=1, \ldots, N_{S}\right)$, and $X_{j}=x_{1}+\cdots+x_{n_{j}}$ is the contribution from the $j^{\text {th }}$ source to the quantity $X$.

Overline notations will be used for the averages connected to a single source. The singlesource quantities are independent of $N_{S}$ and have the properties of intensive quantities. The single-source distribution $F_{S}\left(X_{S}, n\right)$ is assumed to be statistically identical for all sources, thus, for all $j=1, \ldots, N_{S}$ it follows:

$$
\overline{X_{j}^{k}} \equiv \overline{X_{S}^{k}}, \quad \overline{n_{j}^{k}} \equiv \overline{n^{k}}, \quad \overline{X_{j} n_{j}} \equiv \overline{X_{S} n}
$$


where $\overline{X_{S}^{k}}, \overline{n^{k}}$, and $\overline{X_{S} n}$ (for $k=1,2$ ) are the first and second moments of the distribution $F_{S}\left(X_{S}, n\right)$ for a single source. The sources are assumed to be independent. This gives at $i \neq j$ :

$$
\overline{X_{i} X_{j}} \equiv{\overline{X_{S}}}^{2}, \quad \overline{n_{i} n_{j}} \equiv \bar{n}^{2}, \quad \overline{X_{i} n_{j}} \equiv \overline{X_{S}} \bar{n}
$$

Using Eqs. (18) and (19) one finds for the event averages:

$$
\begin{aligned}
& \langle X\rangle=\overline{X_{S}} \cdot\left\langle N_{S}\right\rangle, \quad\left\langle X^{2}\right\rangle=\overline{X_{S}^{2}} \cdot\left\langle N_{S}\right\rangle+{\overline{X_{S}}}^{2}\left[\left\langle N_{S}^{2}\right\rangle-\left\langle N_{S}\right\rangle\right], \\
& \langle N\rangle=\bar{n}\left\langle N_{S}\right\rangle, \quad\left\langle N^{2}\right\rangle=\overline{n^{2}} \cdot\left\langle N_{S}\right\rangle+\bar{n}^{2} \cdot\left[\left\langle N_{S}^{2}\right\rangle-\left\langle N_{S}\right\rangle\right] \\
& \langle X N\rangle=\overline{X_{S} n}\left\langle N_{S}\right\rangle+\overline{X_{S}} \bar{n} \cdot\left[\left\langle N_{S}^{2}\right\rangle-\left\langle N_{S}\right\rangle\right] .
\end{aligned}
$$

A probability distribution $\mathcal{P}_{S}\left(N_{S}\right)$ of the number of sources is needed to calculate $\left\langle N_{S}\right\rangle$ and $\left\langle N_{S}^{2}\right\rangle$ and, in general, it is unknown.

Using Eqs. 20, 22 one obtains:

$$
\begin{aligned}
& \omega[X] \equiv \frac{\left\langle X^{2}\right\rangle-\langle X\rangle^{2}}{\langle X\rangle}=\frac{\overline{X_{S}^{2}}-\bar{X}_{S}^{2}}{\overline{X_{S}}}+\overline{X_{S}} \cdot \frac{\left\langle N_{S}^{2}\right\rangle-\left\langle N_{S}\right\rangle^{2}}{\left\langle N_{S}\right\rangle} \equiv \omega\left[X_{S}\right]+\overline{X_{S}} \cdot \omega\left[N_{S}\right], \\
& \omega[N] \equiv \frac{\left\langle N^{2}\right\rangle-\langle N\rangle^{2}}{\langle N\rangle}=\frac{\overline{n^{2}}-\bar{n}^{2}}{\bar{n}}+\bar{n} \cdot \frac{\left\langle N_{S}^{2}\right\rangle-\left\langle N_{S}\right\rangle^{2}}{\left\langle N_{S}\right\rangle} \equiv \omega[n]+\bar{n} \cdot \omega\left[N_{S}\right],
\end{aligned}
$$

where $\omega\left[X_{S}\right]$ and $\omega[n]$ are the scaled variances for quantities $X_{S}$ and $n$ referring to a single source. The scaled variances $\omega[X]$ and $\omega[N]$ are independent of the average number of sources $\left\langle N_{S}\right\rangle$. Thus, $\omega[X]$ and $\omega[N]$ are intensive quantities. However, they depend on the fluctuations of the number of sources via $\omega\left[N_{S}\right]$ and, therefore, they are not strongly intensive quantities.

From Eqs. 22, 24 it follows:

$$
\begin{aligned}
& \Delta[X, N]=\frac{1}{\omega[x]}\left[\omega\left[X_{S}\right]-\bar{x} \cdot \omega[n]\right] . \\
& \Sigma[X, N]=\frac{1}{\omega[x]}\left[\omega\left[X_{S}\right]+\bar{x} \cdot \omega[n]-2 \frac{\overline{X_{S} n}-\bar{x} \bar{n}^{2}}{\bar{n}}\right],
\end{aligned}
$$

where the relations $\bar{x}=\overline{X_{S}} / \bar{n}=\langle X\rangle /\langle N\rangle$, and the normalization factors 15 have been used.

Note that the terms with $\left\langle N_{S}^{2}\right\rangle$, which are present in the expressions 20 22 for the second moments of $X$ and $N$, are canceled out in the final expressions 25 26). From three second moments $\left\langle X^{2}\right\rangle,\left\langle N^{2}\right\rangle$, and $\langle X N\rangle$ only two linear combinations independent of $\left\langle N_{S}^{2}\right\rangle$ can be constructed. They are defined as the strongly intensive quantities $\Delta$ and $\Sigma$. To remove the 
dependence on $\left\langle N_{S}\right\rangle$, the strongly intensive quantities should be in a form of reducible fractions. This is achieved due to the normalization factors $(15)$.

Only the first and second moments of $X$ and $N$ are required in order to define the strongly intensive quantities $\Delta$ and $\Sigma$. However, in order to calculate the proposed normalization factors $C_{\Sigma}$ and $C_{\Delta}$, additional information is needed, namely the second moment $\overline{x^{2}}$ of single-particle distribution $F(x)$. Note that the first moment $\bar{x}$ can be calculated as $\bar{x}=\langle X\rangle /\langle N\rangle$, and thus to find it the single particle distribution $F(x)$ is not necessarily needed.

The IPM and MIS have similar structure. The difference is that the number of sources $N_{S}$ in the MIS is replaced by the number of particles $N$ in the IPM. Each source can produce many particles, and the number of these particles varies from source to source and from event to event. Besides, the physical quantity $X_{S}$ for particles emitted from the same source may include inter-particle correlations. Therefore, in general, the MIS does not satisfy the assumptions of the IPM. Nevertheless, a formal similarity between the two models can be exploited and gives the following rule of one to one correspondence: all results for the IPM can be found from the expressions obtained within the MIS, assuming artificially that each source always produces exactly one particle. In this case one finds

$$
\bar{n}=1, \quad \omega[n]=0, \quad \omega\left[X_{S}\right]=\omega[x], \quad \overline{X_{S} n}=\bar{x},
$$

and Eqs. 25, 26) are transformed to Eq. (14).

If particles are independently emitted from a single source, one obtains

$$
F_{S}\left(X_{S}, n\right)=\mathcal{P}_{S}(n) \times F_{S}\left(x_{1}\right) \times \cdots \times F_{S}\left(x_{n}\right)
$$

with the probability distributions $F_{S}\left(x_{i}\right)$ which are the same for all $i=1, \ldots, n$ and independent of the number of particles $n$. Similar to Eqs. 10 and (11) one then finds:

$$
\omega\left[X_{S}\right]=\omega[x]+\bar{x} \cdot \omega[n], \quad \overline{X_{S} n}-\overline{X_{S}} \bar{n}=\bar{x} \bar{n} \cdot \omega[n]
$$

and Eqs. 25) and (26) are again transformed to Eq. (14). Therefore, the MIS with independent particle emission from each source is equivalent to the IPM.

Correlations of particles emitted from a single source can be of different origin. Let us consider the case when all single-particle distributions $F_{S}(x)$ in Eq. 28) are dependent on the 
source parameter $T$ (e.g., the source temperature) which fluctuates, and these $T$-fluctuations are independent for each source. The $F_{S}\left(X_{S}, n\right)$ distribution for a single source can be then presented as

$$
F_{S}\left(X_{S}, n\right)=\mathcal{P}_{S}(n) \times \int d T W(T) F_{S}\left(x_{1}, T\right) \times \cdots \times F_{S}\left(x_{n}, T\right)
$$

Note that presentation means the absence of correlations between particle momenta $x_{j}$ and multiplicity $n$, but correlations between $x_{i}$ and $x_{j}$ appear due to the $T$ fluctuations. The multi-particle distribution (30) may look as a simple product of the one-particle distributions. However, the single particle distributions are not independent due to integration over $T$. With distribution (30) one calculates

$$
\begin{aligned}
\overline{X_{S} n} & =\sum_{n} \mathcal{P}_{S}(n) \int d T W(T) \int d x_{1} \ldots d x_{n} F_{S}\left(x_{1}, T\right) \ldots F_{S}\left(x_{n}, T\right)\left(x_{1}+\cdots+x_{n}\right) \cdot n \\
& =\bar{x} \overline{n^{2}} .
\end{aligned}
$$

Using Eq. (31) one can simplify further Eq. (26). Finally, it gives:

$$
\Delta[X, N]=\Sigma[X, N]=\frac{\omega\left[X_{S}\right]-\bar{x} \cdot \omega[n]}{\omega[x]} .
$$

\section{FAST GENERATORS AND ANALYTICAL RESULTS}

We consider the Boltzmann transverse momentum $\left(p \equiv p_{T}\right)$ distribution

$$
f(p, T)=C p \exp \left(-\frac{\sqrt{m^{2}+p^{2}}}{T}\right)
$$

where constant $C$ is defined by the normalization condition and $C^{-1}=$ $\int_{0}^{\infty} d p p \exp \left(-\sqrt{m^{2}+p^{2}} / T\right)$. The particle mass $m$ in the MC simulations is taken as the pion mass $m=m_{\pi} \cong 140 \mathrm{MeV}, T$ is the effective temperature or simply an inverse slope parameter controlled by the actual freeze-out temperature and the collective transverse flow velocity. The moments $(k=1,2)$ of the $f(p, T)$ probability distributions (33) are denoted as

$$
\tilde{p^{k}}=\int_{0}^{\infty} d p p^{k} f(p, T) .
$$


In the presence of e-by-e temperature fluctuations, the inclusive transverse momentum distributions reads

$$
f(p)=\int d T W(T) f(p, T)
$$

where $W(T)$ is the temperature probability distribution normalized to one. The moments $(k=1,2)$ of the $f(p)$ probability distribution $(35)$ are denoted as

$$
\overline{p^{k}}=\int_{0}^{\infty} d p p^{k} f(p)=\int d T W(T) \tilde{p^{k}}
$$

In the case of massless particles $m=0$ distribution $(33)$ is reduced to a simple exponential form and one can easily compute

$$
\tilde{p}=2 T, \quad \tilde{p^{2}}=6 T^{2}, \quad \bar{p}=2 \bar{T}, \quad \overline{p^{2}}=6 \overline{T^{2}}, \quad \omega[p]=\frac{3 \overline{T^{2}}-2 \bar{T}^{2}}{\bar{T}},
$$

where $(k=1,2)$

$$
\overline{T^{k}}=\int d T T^{k} W(T)
$$

Note that in the MC simulations the particle transverse momenta are generated with the $p$ values in a region $\left[0, p_{\max }\right]$.

The basic properties of $\Delta\left[P_{T}, N\right]$ and $\Sigma\left[P_{T}, N\right]$ measures will be tested using MC simulations (so-called fast generators). Each interaction (event) is composed by a given number of sources. For each simulation the statistical errors on $\Delta\left[P_{T}, N\right]$ and $\Sigma\left[P_{T}, N\right]$ are estimated as follows. The whole sample of events is divided into 30 independent sub-samples. Next, the values of $\Delta\left[P_{T}, N\right]$ and $\Sigma\left[P_{T}, N\right]$ are evaluated for each sub-sample and the dispersions $\left(D_{\Delta}\right.$, and $\left.D_{\Sigma}\right)$ of the results are then calculated. The statistical error of $\Delta\left[P_{T}, N\right]$ or $\Sigma\left[P_{T}, N\right]$ is taken to be equal to $D_{\Delta} / \sqrt{30}$ or $D_{\Sigma} / \sqrt{30}$, respectively.

\section{A. Fixed Temperature}

The first set of the MC simulations refers to particle production from sources with fixed temperature. For each source in a given event the number of particles was generated from the Poisson distribution with a mean value of 5 . The particle transverse momentum was generated 

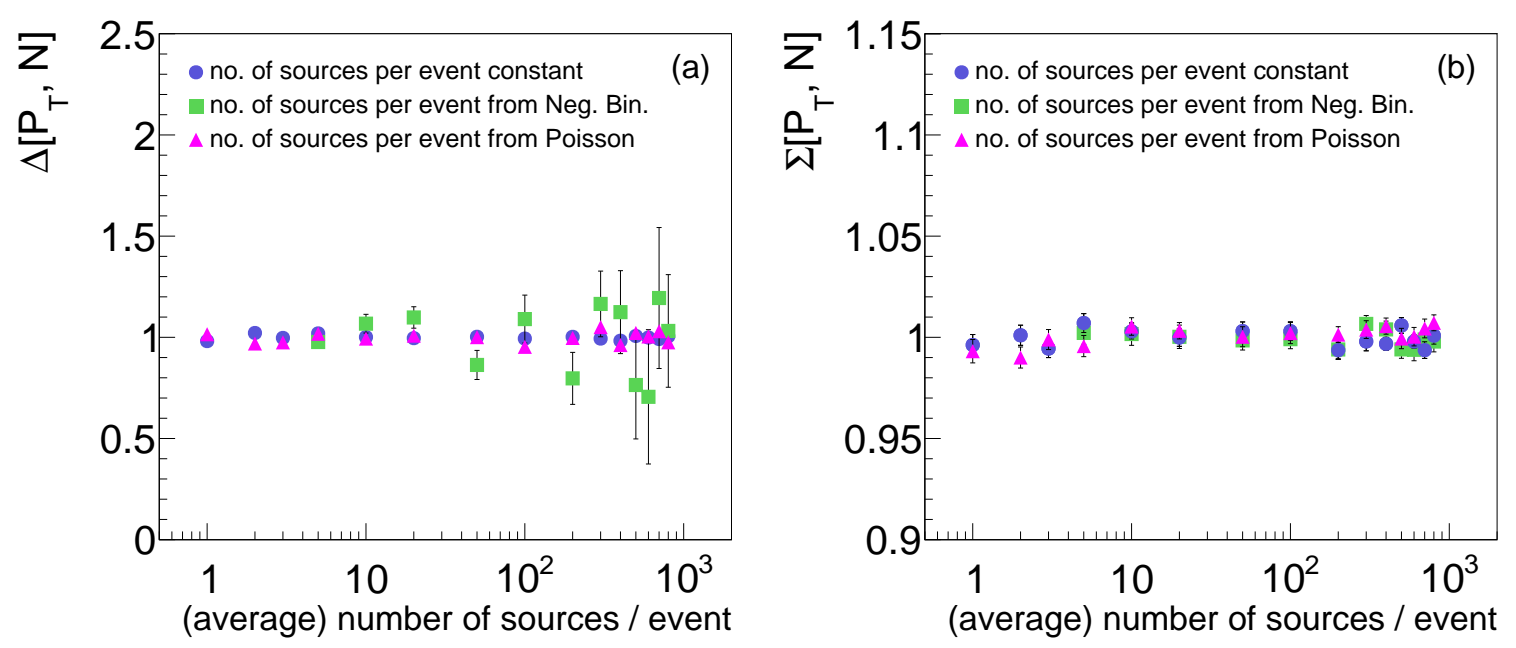

Figure 1: (Color online) The symbols are the MC results for the $\Delta\left[P_{T}, N\right](a)$ and $\Sigma\left[P_{T}, N\right](b)$ measures versus the number or mean number of sources composing one event. All sources have fixed temperature. The number of sources per event are fixed (circles) or fluctuating according to the Poisson distribution (triangles) and Negative Binomial distribution (squares).

from transverse momentum distribution (33) with maximal value $p_{\max }=2.0 \mathrm{GeV} / c$. The temperature parameter is fixed as $T=150 \mathrm{MeV}$. The number of sources $N_{S}$ composing an event is either constant (circles in Fig. 1) or selected from Poisson (triangles) or from Negative Binomial distribution (squares). For Negative Binomial distribution its dispersion $\sqrt{\operatorname{Var}\left(N_{S}\right)}$ is large and equals $\left\langle N_{S}\right\rangle / 2$.

Figure 1 shows $\Delta\left[P_{T}, N\right]$ and $\Sigma\left[P_{T}, N\right]$ versus the number or the mean number of sources composing one event. The distribution function of a single source has the form of Eq. (28) and the $P_{S}(x)$ function is taken as $f(p, T)$ (33) with fixed temperature $T$, same for all sources. This corresponds the case when the MIS is reduced to the IPM, and relation (14) should be valid. As expected, the $\Delta\left[P_{T}, N\right]$ and $\Sigma\left[P_{T}, N\right]$ values for the $\mathrm{MC}$ simulations are consistent with one, independently of the assumed shape of transverse momentum distribution. The circles in Fig. 1 confirm that $\Delta\left[P_{T}, N\right]$ and $\Sigma\left[P_{T}, N\right]$ are intensive measures (do not depend on $N_{S}$ ), whereas the triangles and the squares show that these quantities are also strongly intensive (do not depend on $N_{S}$ fluctuations). 
For a constant number of sources per event (circles in Fig. 1), the scaled variance of multiplicity distribution $\omega[N]=\omega[n]+\bar{n} \omega\left[N_{S}\right]$ equals 1 in the whole range of the horizontal axis. For the Poisson distribution of the number of sources $\omega[N]$ equals to 6 also for the whole range of the mean number of sources per event. For the Negative Binomial distribution of the number of sources $\omega[N]$ increases from about 7 at $\left\langle N_{S}\right\rangle=5$, through 126 at $\left\langle N_{S}\right\rangle=100$, up to approximately 1000 at $\left\langle N_{S}\right\rangle=800$. Therefore, Fig. 1 shows that $\Delta\left[P_{T}, N\right]$ and $\Sigma\left[P_{T}, N\right]$ measures are strongly intensive even for multiplicity distributions which are extremely wide.

\section{B. Source Temperature Fluctuations}

In the next set of simulations, the number of particles produced by each single source is again selected from the Poisson distribution with a mean value of $\bar{n}=5$. The particle transverse momentum is generated by the transverse momentum distribution (33) with average inverse slope parameter $\bar{T}=150 \mathrm{MeV}$. The $T$ parameter is generated separately for each single source (source-by-source $T$ fluctuations) from the Gaussian distribution

$$
W(T)=\frac{1}{\sqrt{2 \pi} \sigma_{T}} \exp \left[-\frac{(T-\bar{T})^{2}}{2 \sigma_{T}^{2}}\right]
$$

with dispersion $\sigma_{T}=25 \mathrm{MeV}$. Finally, the number of sources $N_{S}$ composing an event is generated from the Poisson distribution, with $\left\langle N_{S}\right\rangle$ as denoted on the horizontal axis of Fig. 2 . As seen, the effect of source temperature fluctuations results in $\Delta\left[P_{T}, N\right]$ and $\Sigma\left[P_{T}, N\right]$ values higher than 1.

If the parameter $T$ fluctuates independently for each source, the sources remain to be statistically identical and independent of each other. Therefore, these MC simulations correspond to the MIS and the $\Delta\left[P_{T}, N\right]$ and $\Sigma\left[P_{T}, N\right]$ strongly intensive measures should not depend on the mean number of sources $\left\langle N_{S}\right\rangle$ and on its fluctuations $\omega\left[N_{S}\right]$. Indeed, Fig. 2 confirms this expectation.

The distribution function of a single source has the form of Eq. (30) with $F_{S}(x, T)$ function taken as $f(p, T)$. This leads to the result $\left(P \equiv P_{T}\right)(32)$

$$
\Delta[P, N]=\Sigma[P, N]=\frac{\omega\left[P_{S}\right]-\bar{p} \cdot \omega[n]}{\omega[p]}, \quad \omega[p] \equiv \frac{\overline{p^{2}}-\bar{p}^{2}}{\bar{p}} .
$$




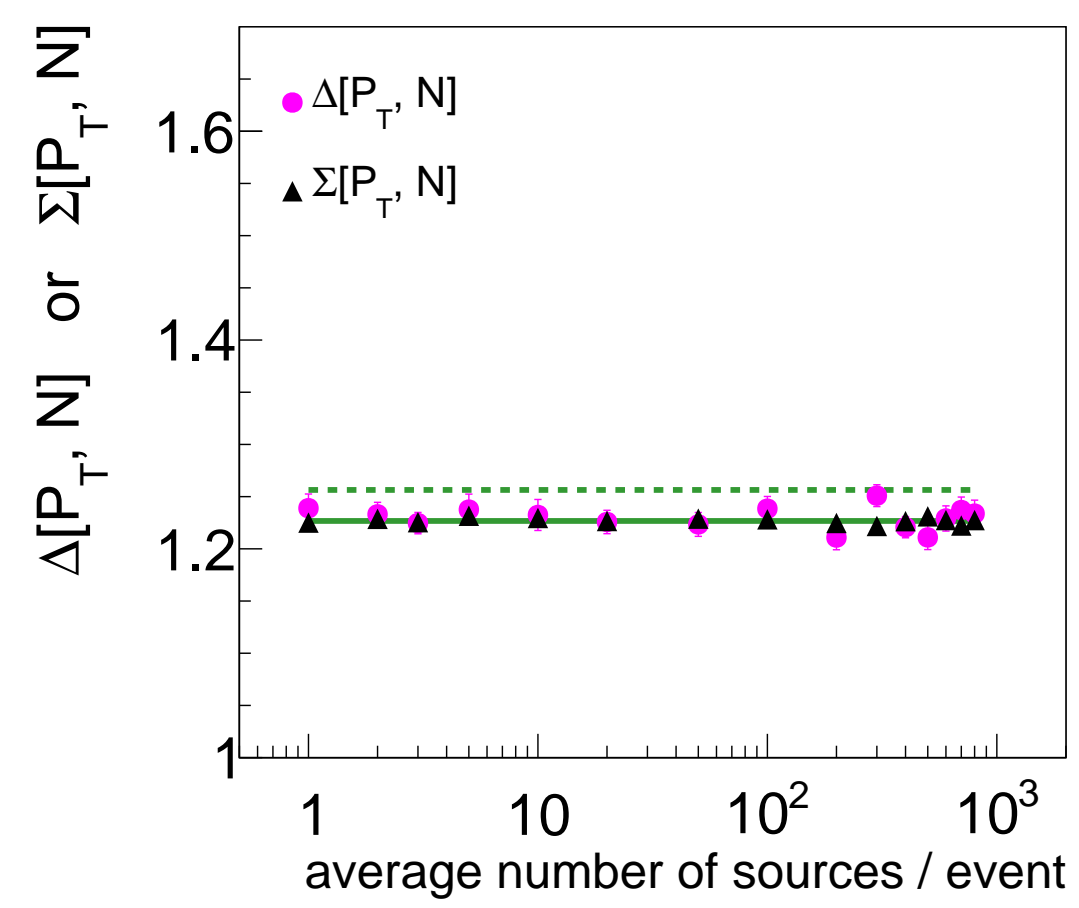

Figure 2: (Color online) The symbols are the MC results for the $\Delta\left[P_{T}, N\right]$ (circles) and $\Sigma\left[P_{T}, N\right]$ (triangles) measures versus the mean number of sources composing one event. The temperatures of the sources fluctuate independently according to Eq. (39), and the number of sources per event fluctuates according to the Poisson distribution. The solid line corresponds to Eq. (48), the dashed line to Eq. (51).

For $\overline{P_{S}}$ and $\overline{P_{S}^{2}}$ one obtains:

$$
\begin{aligned}
& \overline{P_{S}}=\bar{p} \cdot \bar{n} \\
& \overline{\left(P_{S}\right)^{2}}=\sum_{n} \mathcal{P}_{S}(n) \int d T W(T) \int_{0}^{\infty} \prod_{i=1}^{n}\left[p_{i} d p_{i} f\left(p_{i}\right)\right]\left(p_{1}+\ldots+p_{n}\right)^{2} \\
& =\sum_{n} \mathcal{P}_{S}(n) \int d T W(T) \int_{0}^{\infty} \prod_{i=1}^{n}\left[p_{i} d p_{i} f\left(p_{i}\right)\right]\left[\sum_{j=1}^{n} p_{j}^{2}+\sum_{1 \leq l \neq m \leq k} p_{l} \cdot p_{m}\right] \\
& =\sum_{n} \mathcal{P}_{S}(k) \int d T W(T)\left[n \cdot \tilde{p^{2}}+n(n-1) \cdot \tilde{p}^{2}\right]=\overline{p^{2}} \cdot \bar{n}+\hat{p}^{2} \cdot\left[\overline{n^{2}}-\bar{n}\right],
\end{aligned}
$$


where

$$
\hat{p}^{2} \equiv \int d T W(T) \tilde{p}^{2}
$$

Calculating $\omega\left[P_{S}\right]$ from Eqs. 41 42 and inserting it into Eq. 32 one obtains:

$$
\Delta[P, N]=\Sigma[P, N]=1+\frac{1}{\omega[p]} \cdot \frac{\hat{p}^{2}-\bar{p}^{2}}{\bar{p}} \cdot[\bar{n}+\omega[n]-1] .
$$

One can easily prove that

$$
\begin{aligned}
& \hat{p}^{2}-\bar{p}^{2}=\int d T W(T)(\tilde{p}-\bar{p})^{2} \geq 0, \\
& \bar{n}+\omega[n]-1=\frac{\overline{n^{2}}-\bar{n}}{\bar{n}}=\frac{1}{\bar{n}} \sum_{n \geq 2} \mathcal{P}_{S}(n)\left(n^{2}-n\right) \geq 0 .
\end{aligned}
$$

When temperature fluctuations are absent, relation 45 is transformed to $\hat{p}^{2}-\bar{p}^{2}=0$, and Eq. (44) is reduced to Eq. (14). The same happens when $\mathcal{P}_{S}(n)=0$ for all $n \geq 2$, and, thus, $\bar{n}+\omega[n]-1=0$. This is intuitively clear: the MIS is reduced to the IPM if each source can emit only one or zero number of particles.

In Fig. 2 the results of the MC simulations are compared with analytical results of Eq. (44). The solid line corresponds to the distribution (33) and Gaussian temperature fluctuations (39). In this case one finds:

$$
\bar{p} \cong 0.328 \mathrm{GeV} / c, \quad \overline{p^{2}} \cong 0.158(\mathrm{GeV} / c)^{2}, \quad \hat{p}^{2} \cong 0.110(\mathrm{GeV} / c)^{2}
$$

The $\mathcal{P}_{S}(n)$ Poisson distribution for a single source corresponds to $\bar{n}=5$ and $\omega[n]=1$, therefore, $\bar{n}+\omega[n]-1=5$. The final result of Eq. (44) is

$$
\Delta[P, N]=\Sigma[P, N] \cong 1.227
$$

As seen in Fig. 2, this is in a good agreement with the results of the MC simulations.

For massless particles the quantities in Eq. (47) can be calculated analytically

$$
\begin{aligned}
& \bar{p}=2 \bar{T}=0.3 \mathrm{GeV} / c, \quad \overline{p^{2}}=6 \overline{T^{2}}=6\left[\bar{T}^{2}+\sigma_{T}^{2}\right]=0.13875(\mathrm{GeV} / c)^{2}, \\
& \hat{p^{2}}=\int d T W(T) \tilde{p}^{2}=4 \overline{T^{2}}=4\left[\bar{T}^{2}+\sigma_{T}^{2}\right]=0.0925(\mathrm{GeV} / c)^{2} .
\end{aligned}
$$


With Eqs. 49 50 one finds

$$
\Delta[P, N]=\Sigma[P, N]=1+\frac{2 \sigma_{T}^{2}}{\bar{T}^{2}+3 \sigma_{T}^{2}} \cdot[\bar{n}+\omega[n]-1] .
$$

For the values $\bar{T}=0.15 \mathrm{GeV}, \sigma_{T}=0.025 \mathrm{GeV}, \bar{n}=5$, and $\omega[n]=1$ used in the MC simulations one finds $\Delta[P, N]=\Sigma[P, N] \cong 1.256$. This result for $m=0$ is shown in Fig. 2 by the dashed line.

The MC results presented in Figs. 1 and 2 demonstrate a different sensitivity of the strongly intensive measures to model details: despite of the equality $\Delta\left[P_{T}, N\right]=\Sigma\left[P_{T}, N\right]$ the statistical errors of the simulations calculated for $\Delta\left[P_{T}, N\right]$ are found to be essentially larger than those for $\Sigma\left[P_{T}, N\right]^{1}$.

\section{Global Temperature Fluctuations}

In the next MC simulations, source-by-source $T$ fluctuations from the previous subsection are replaced by e-by-e (global) $T$ fluctuations. The parameter $T$ is the same for all sources composing a given event but is varied between events following the Gaussian distribution (39) with average inverse slope parameter $\langle T\rangle=150 \mathrm{MeV}$ and dispersion $\sigma_{T}$. The number of sources $N_{S}$ composing an event is generated from the Poisson distribution with $\left\langle N_{S}\right\rangle$ being the average value. As previously, for each single source, the number of particles was selected from the Poisson distribution with a mean value of $\bar{n}=5$. The results are presented in Fig. 3. Its (a) panel shows the dependence of $\Delta\left[P_{T}, N\right]$ and $\left.\Sigma\left[P_{T}, N\right]\right)$ on the average number of sources $\left\langle N_{S}\right\rangle$ at $\sigma_{T}=25 \mathrm{MeV}$, whereas the (b) panel presents the dependence on $\sigma_{T}$ at $\left\langle N_{S}\right\rangle=100$. In Fig. 3 (b) in order to avoid negative $T$ values only events within $T=150 \pm 3 \sigma_{T} \mathrm{MeV}$ were accepted. We also would like to mention here that the relationship between temperature and multiplicity (or volume) fluctuations was studied in Refs. [37, 38].

Due to the correlated $T$-fluctuations for different sources, the sources are not independent of each other. Therefore, these MC simulations do not correspond to the MIS. One can never-

\footnotetext{
${ }^{1}$ In order to avoid too large statistical errors, in Fig. 2 we used five times higher statistics (500k events for each point) than that one used in Figs. 1 and 3
} 

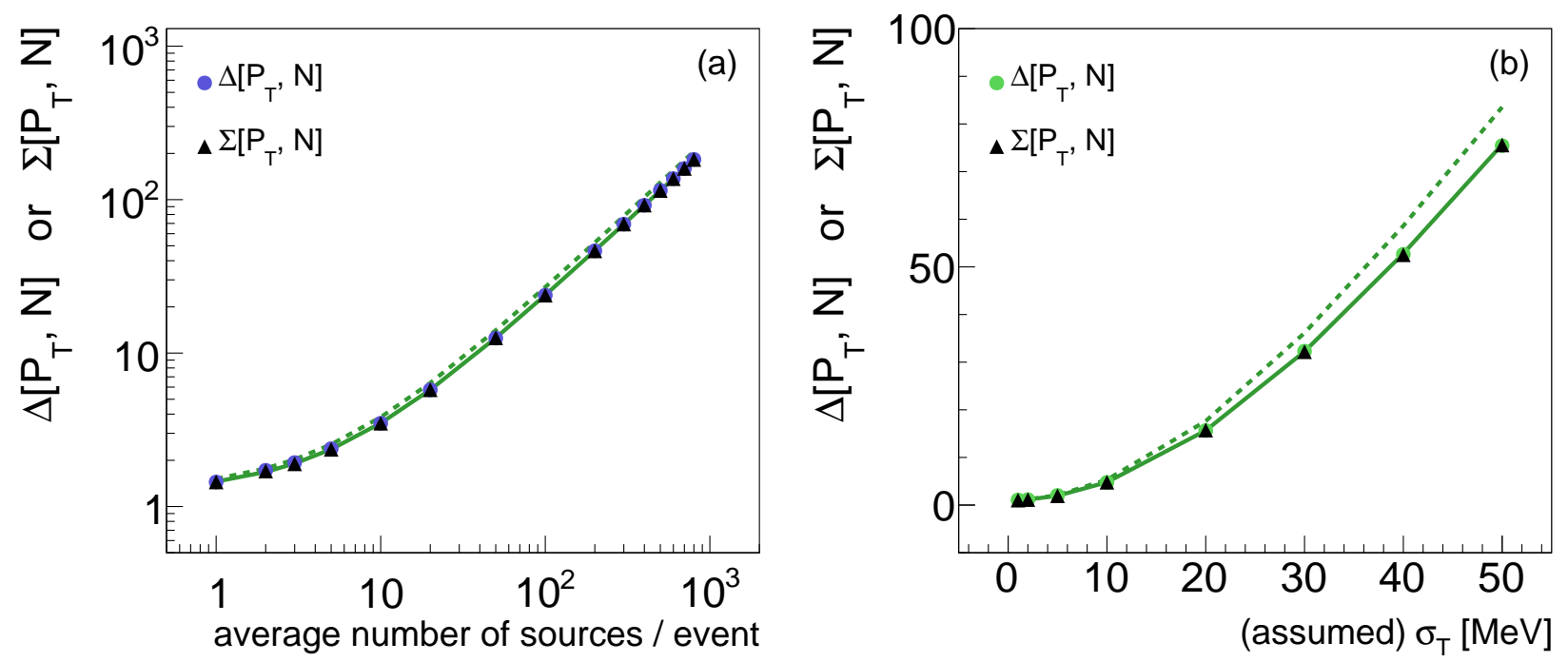

Figure 3: (Color online) The symbols are the MC results for the $\Delta\left[P_{T}, N\right]$ (circles) and $\Sigma\left[P_{T}, N\right]$ (triangles) measures. The MC simulations correspond to the global temperature fluctuations according to Eq. (39), i.e. temperatures of all sources are equal. The number of sources are taken from the Poisson distribution with the average value of $\left\langle N_{S}\right\rangle$. The solid lines present the results of Eq. (53), the dashed lines of Eq. (55). (a): The dependence on the mean number of sources at fixed $\sigma_{T}=25 \mathrm{MeV}$. (b): The dependence on $\sigma_{T}$ at fixed $\left\langle N_{S}\right\rangle=100$ (here for calculating dashed line the obtained $\sigma_{T}$ values were used; due to the limited range of $T$ distribution they are slightly smaller than the assumed ones).

theless use the formula from the previous subsection with the following substitutions:

$$
N_{S} \rightarrow 1, \quad \omega\left[N_{S}\right] \rightarrow 0, \quad n \rightarrow N, \quad P_{S} \rightarrow P
$$

i.e. all final particles are treated as created from a "single source" with fluctuating temperature $T$. Note that the parameter $T$ becomes an event variable with average value $\langle T\rangle=\bar{T}=$ $150 \mathrm{MeV}$ and distribution (39). This gives:

$$
\Delta[P, N]=\Sigma[P, N]=1+\frac{1}{\omega[p]} \cdot \frac{\hat{p}^{2}-\bar{p}^{2}}{\bar{p}} \cdot[\langle N\rangle+\omega[N]-1] .
$$

The MC results on global temperature fluctuations are compared to analytical predictions of Eq. (53). The solid lines in Fig. 3 correspond to the transverse momentum distribution 
(33) with temperature fluctuating according to Eq. (39). The values of $\bar{p}, \overline{p^{2}}, \hat{p}^{2}$, and $\omega[p]$ are calculated numerically with Eqs. (33) and (39). At $\sigma_{T}=25 \mathrm{MeV}$, they are equal to those in Eq. (47). Analytical calculations can be done for massless particles according to Eq. (49) which demonstrates the explicit dependence on $\sigma_{T}$.

Note that multiplicities $n_{1}, \ldots, n_{N_{S}}$ for particles emitted by different sources are uncorrelated. Therefore, one can use the MIS to calculate $\langle N\rangle$ and $\omega[N]$ with Eq. (24):

$$
\langle N\rangle=\bar{n}\left\langle N_{S}\right\rangle=5\left\langle N_{S}\right\rangle, \quad \omega[N]=\omega[n]+\bar{n} \cdot \omega\left[N_{S}\right]=1+5 \cdot 1=6
$$

This results in a linear increase of $(53)$ with $\left\langle N_{S}\right\rangle$.

For $m=0$ in the distribution (33), similarly to Eq. (51), one obtains

$$
\Delta[P, N]=\Sigma[P, N]=1+\frac{2 \sigma_{T}^{2}}{\langle T\rangle^{2}+3 \sigma_{T}^{2}} \cdot\left[5\left\langle N_{s}\right\rangle+5\right]
$$

where Eq. (54) has been already used. This is shown in Fig. 3 by dashed lines.

As expected from Eq. (53), the fluctuation measures $\Delta\left[P_{T}, N\right]$ and $\left.\Sigma\left[P_{T}, N\right]\right)$ increase when global temperature fluctuations are stronger (higher $\sigma_{T}$ ). This is explicitly seen from Eq. (55) for $m=0$. The same conclusion was drawn in Ref. [25], where the influence of temperature fluctuations on transverse momentum fluctuations was studied for the $\Phi_{p_{T}}$ measure [9] (see also Ref. [7] for the corresponding plot).

\section{TEMPERATURE CORRELATIONS VERSUS NUMBER OF PARTICLES}

The results of fast generators in the previous section showed the same behavior and magnitudes of $\Delta\left[P_{T}, N\right]$ and $\Sigma\left[P_{T}, N\right]$ measures. The MC simulation, presented in this section, is introduced in order to check whether one can propose a fast generator for which different values of $\Delta\left[P_{T}, N\right]$ and $\Sigma\left[P_{T}, N\right]$ may be obtained. As an example, we consider the $M\left(p_{T}\right)$ versus $N$ correlation suggested in Ref. [9], where $M\left(p_{T}\right)$ is the event mean single-particle transverse momentum and $N$ is the particle multiplicity. In Fig. 4 (a) the assumed multiplicity distribution is presented as red triangles (those values correspond to the accepted multiplicities at forward rapidities in $p+p$ collisions at the beam energy $158 \mathrm{GeV}$ [11]). As seen, the generated multiplicity distribution (gray histogram) coincides with the assumed one. For each event, 
particle momenta are generated from transverse momentum distribution (33) with $T$ taken as $T_{N}=\left\langle M\left(p_{T}\right)\right\rangle_{N} / 2$, where $\left\langle M\left(p_{T}\right)\right\rangle_{N}$ is dependent on generated multiplicity $N$ as shown in Fig. 4 (b) by the red triangles [11]. The range of $p_{T}$ generation is from zero to $2 \mathrm{GeV} / c$. In Fig. $4(b)$ the scatter plot represents all generated events $\left(M\left(p_{T}\right)\right.$ values) and the gray squares their profile histogram $\left(\left\langle M\left(p_{T}\right)\right\rangle_{N}\right.$ values, where $\langle\ldots\rangle_{N}$ represents averaging within the same multiplicity $N$ ). The difference between red triangles (input values of $2 T_{N}$ used in simulation) and gray squares $\left(\left\langle M\left(p_{T}\right)\right\rangle_{N}\right.$ values obtained from simulated data set) is due to the fact that in transverse momentum distribution used in simulation (33) the average transverse momentum is only approximately equal to $2 T$. It was, however, verified by an independent analysis that when using $f(p, T)=C p \exp (-p / T)$ distribution, for which the mean transverse momentum equals exactly $2 T$, red and gray points coincide. For the simulation presented in Fig. 4 the values of fluctuation measures obtained for 500000 generated events are:

$$
\Delta\left[P_{T}, N\right]=0.8158 \pm 0.0051, \quad \Sigma\left[P_{T}, N\right]=1.0075 \pm 0.0018
$$

Particle production considered in this section corresponds to the distribution

$$
F_{N}\left(p_{1}, \ldots, p_{N}\right)=\mathcal{P}(N) \times f_{N}\left(p_{1}\right) \times \cdots \times f_{N}\left(p_{N}\right)
$$

where $f_{N}(p)=f\left(p, T_{N}\right)$ with $f\left(p, T_{N}\right)$ given by Eq. (33), but with the parameter $T$ depending now on the particle multiplicity, $T_{N}=T(N)$. The moments of single particle distributions at fixed $N$ are then equal to

$$
\left(\overline{p^{k}}\right)_{N}=\int_{0}^{\infty} d p p^{k} f_{N}(p)
$$

The moments of single particle spectrum averaged over $N$ are

$$
\overline{p^{k}}=\sum_{N} \frac{N \mathcal{P}(N)}{\langle N\rangle}\left(\overline{p^{k}}\right)_{N},
$$

and $\omega[p]=\left(\overline{p^{2}}-\bar{p}^{2}\right) / \bar{p}$.

With distribution (57) one finds:

$$
\begin{aligned}
& \langle P\rangle=\sum_{N} \mathcal{P}(N) \int f_{N}\left(p_{1}\right) d p_{1} \cdots \int f_{N}\left(p_{N}\right) d p_{N}\left(p_{1}+\cdots+p_{N}\right) \\
& =\sum_{N} \mathcal{P}(N) N \int d p f_{N}(p) p=\sum_{N} \mathcal{P}(N) N(\bar{p})_{N}=\bar{p} \cdot\langle N\rangle
\end{aligned}
$$



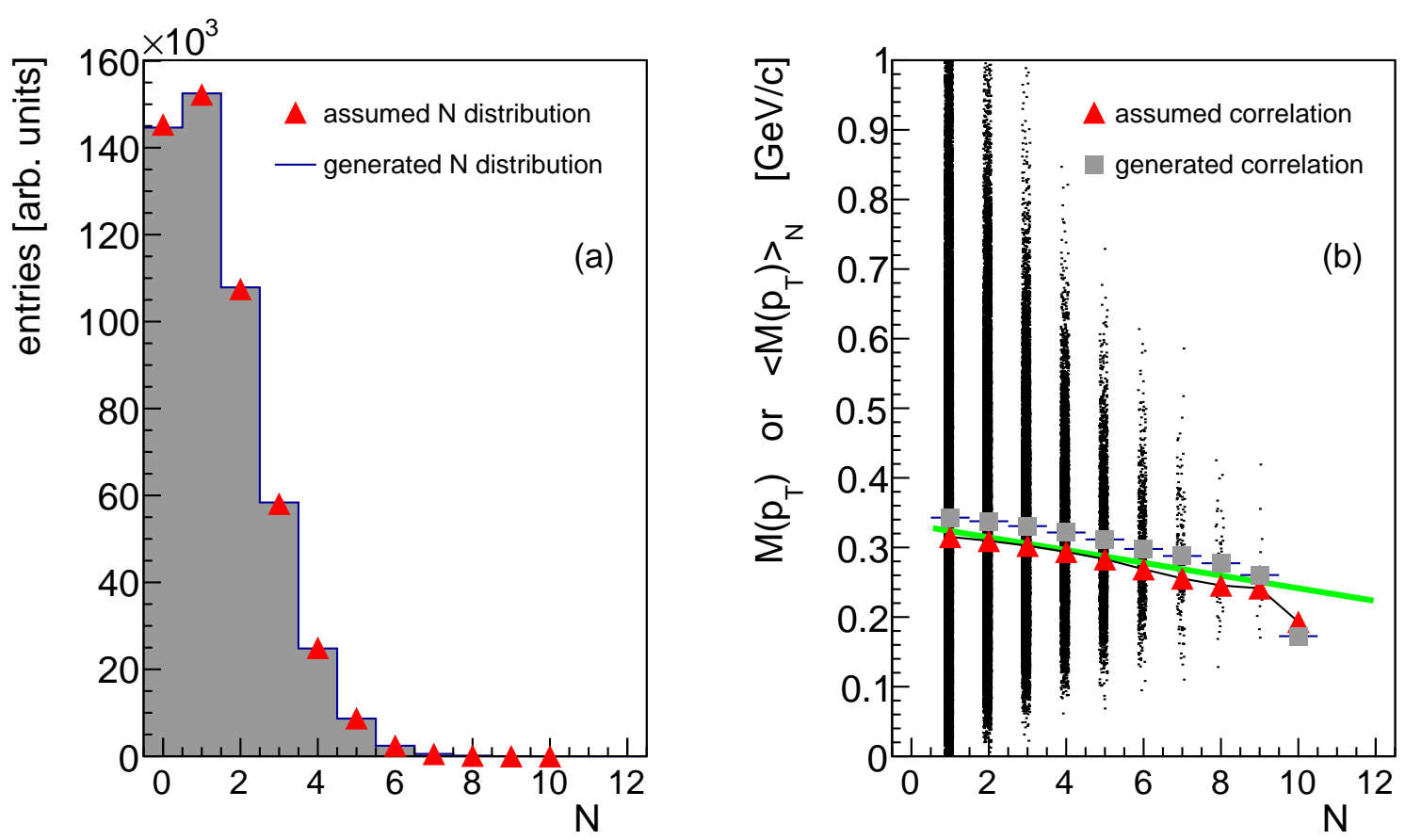

Figure 4: (Color online) Properties of the fast generator producing $M\left(p_{T}\right)$ versus $N$ correlation (see the text for details). Green solid line (b) shows Eqs. (69, 70), where $\langle N\rangle=1.4$ and $T_{N}=\left\langle M\left(p_{T}\right)\right\rangle_{N} / 2$.

$$
\begin{aligned}
& \left\langle P^{2}\right\rangle=\sum_{N} \mathcal{P}(N) \int f_{N}\left(p_{1}\right) d p_{1} \cdots \int f_{N}\left(p_{N}\right) d p_{N}\left(p_{1}+\cdots+p_{N}\right)^{2} \\
& =\sum_{N} \mathcal{P}(N)\left[N \int d p f_{N}(p) p^{2}+N(N-1) \cdot\left(\int d p f_{N}(p) p\right)^{2}\right] \\
& =\sum_{N} \mathcal{P}(N)\left[N \cdot\left(\overline{p^{2}}\right)_{N}+N(N-1) \cdot(\bar{p})_{N}^{2}\right] \\
& =\left[\overline{p^{2}}-\bar{p}^{2}\right] \cdot\langle N\rangle+\sum_{N} \mathcal{P}(N) N^{2}(\bar{p})_{N}^{2} \\
& \langle P N\rangle=\sum_{N} \mathcal{P}(N) N \int f_{N}\left(p_{1}\right) d p_{1} \cdots \int f_{N}\left(p_{N}\right) d p_{N}\left(p_{1}+\cdots+p_{N}\right) \\
& =\sum_{N} \mathcal{P}(N) N^{2}(\bar{p})_{N} .
\end{aligned}
$$


This gives:

$$
\begin{aligned}
& \omega[P]=\omega[p]+\frac{\sum_{N} \mathcal{P}(N) N^{2} \cdot(\bar{p})_{N}^{2}-\bar{p}^{2} \cdot\langle N\rangle^{2}}{\bar{p} \cdot\langle N\rangle} \\
& \langle P N\rangle-\langle P\rangle\langle N\rangle=\sum_{N} \mathcal{P}(N) \cdot N^{2} \cdot(\bar{p})_{N}-\bar{p} \cdot\langle N\rangle^{2} .
\end{aligned}
$$

Finally,

$$
\begin{aligned}
& \Delta[P, N]=1+\frac{\bar{p}}{\omega[p] \cdot\langle N\rangle} \sum_{N} \mathcal{P}(N) N^{2}\left(Y_{N}^{2}-1\right) \\
& \Sigma[P, N]=1+\frac{\bar{p}}{\omega[p] \cdot\langle N\rangle} \sum_{N} \mathcal{P}(N) N^{2}\left(Y_{N}-1\right)^{2}
\end{aligned}
$$

where $Y_{N} \equiv(\bar{p})_{N} / \bar{p}$. Calculating numerically 65 and 66 with $\mathcal{P}(N)$ and $T_{N}=\left\langle M\left(p_{T}\right)\right\rangle_{N} / 2$ presented in Fig. $4(a)$ and $(b)$, respectively, one finds the $\Delta\left[P_{T}, N\right]$ and $\Sigma\left[P_{T}, N\right]$ values which coincide with those in Eq. (56) within statistical uncertainties.

To make further analytical calculations several simplifying assumptions will be adopted. First, it will be assumed that produced particles are massless. For $m=0$ in the distribution (33) with $T=T_{N}$ one finds $(\bar{p})_{N}=2 T_{N},\left(\overline{p^{2}}\right)_{N}=6 T_{N}^{2}$, and

$$
\begin{aligned}
\bar{p} & =\frac{1}{\langle N\rangle} \sum_{N} N \mathcal{P}(N)(\bar{p})_{N}=2 T\left[1+\theta\left(1-\frac{\left\langle N^{2}\right\rangle}{\langle N\rangle^{2}}\right)\right] \\
\overline{p^{2}} & =\frac{1}{\langle N\rangle} \sum_{N} N \mathcal{P}(N)\left(\overline{p^{2}}\right)_{N}=\frac{6 T^{2}}{\langle N\rangle} \sum_{N} N \mathcal{P}(N)\left[1+\theta \cdot\left(1-\frac{N}{\langle N\rangle}\right)\right]^{2} \\
& =6 T^{2}\left[1-\theta \cdot \frac{\left\langle N^{2}\right\rangle}{\langle N\rangle^{2}}+\theta^{2} \cdot\left(1-2 \frac{\left\langle N^{2}\right\rangle}{\langle N\rangle^{2}}+\frac{\left\langle N^{3}\right\rangle}{\langle N\rangle^{3}}\right)\right] .
\end{aligned}
$$

Second, a parametrization for the multiplicity dependent temperature

$$
T_{N}=T\left[1+\theta \cdot\left(1-\frac{N}{\langle N\rangle}\right)\right]
$$

proposed in Ref. [29] will be adopted. This formula, with small positive dimensionless parameter $\theta$, is approximately valid for the data in $p+p$ collisions at SPS energy presented in Fig. 4 ( $(b)$. Using the value of $\langle N\rangle=1.4$ (found from the data in Fig. $4(a)$ ) the values of

$$
T \cong 160 \mathrm{MeV}, \quad \theta \cong 0.04
$$

are fixed from fitting the data in Fig. $4(b)$. The correlation of the inverse slope ('temperature') parameter $T_{N}$ versus $N$ in a form of Eq. (69) with $\theta>0$ is probably of simple kinematic 
origin: when the multiplicity of produced particles increases at fixed collision energy, there is less and less energy to be transformed to transverse momenta of produced particles. As a result, the average transverse momentum per particle decreases when $N$ grows. However, in $\mathrm{A}+\mathrm{A}$ collisions the contribution of the transverse collective flow to particle transverse momenta becomes important. This collective flow, in its turn, increases with the number of produced particles. Therefore, a correlation between $T_{N}$ and $N$ in a form (69), but with $\theta<0$, may be expected.

For further calculations we make the third simplification assuming the Poisson shape for $\mathcal{P}(N)$ distribution. In this case one obtains

$$
\begin{aligned}
& \left\langle N^{2}\right\rangle=\langle N\rangle^{2}+\langle N\rangle, \quad\left\langle N^{3}\right\rangle=\langle N\rangle^{3}+3\langle N\rangle^{2}+\langle N\rangle \\
& \left\langle N^{4}\right\rangle=\langle N\rangle^{4}+6\langle N\rangle^{3}+7\langle N\rangle^{2}+\langle N\rangle
\end{aligned}
$$

and Eqs. 67) and (68) are transformed to

$$
\bar{p}=2 T\left[1-\frac{\theta}{\langle N\rangle}\right], \quad \overline{p^{2}}=6 T^{2}\left[1-\frac{2 \theta}{\langle N\rangle}+\theta^{2} \cdot\left(\frac{1}{\langle N\rangle}+\frac{1}{\langle N\rangle^{2}}\right)\right] .
$$

This gives

$$
\omega[p]=\frac{\overline{p^{2}}-\bar{p}^{2}}{\bar{p}} \cong T\left[1-\frac{\theta}{\langle N\rangle}\right], \quad Y_{N}=\frac{(\bar{p})_{N}}{\bar{p}} \cong 1+\theta \cdot\left[1-\frac{N}{\langle N\rangle}\right]
$$

where the second and higher powers of $\theta$ have been neglected and $\langle N\rangle \gg 1$ is assumed (this is our fourth and the last simplification). For $\Sigma[P, N]$ (66) one obtains

$$
\begin{aligned}
& \Sigma[P, N]=1+\frac{\bar{p}}{\omega[p]\langle N\rangle} \sum_{N} \mathcal{P}(N) N^{2}\left[Y_{N}-1\right]^{2} \\
& \cong 1+2 \frac{\theta^{2}}{\langle N\rangle}\left[\left\langle N^{2}\right\rangle-2 \frac{\left\langle N^{3}\right\rangle}{\langle N\rangle}+\frac{\left\langle N^{4}\right\rangle}{\langle N\rangle^{2}}\right]=1+2 \theta^{2},
\end{aligned}
$$

where Eqs. (71) and (72) have been used at the last step in Eq. (75).

The $\Delta[P, N](65)$ is calculated as

$$
\begin{aligned}
& \Delta[P, N]=1+\frac{\bar{p}}{\omega[p] \cdot\langle N\rangle} \sum_{N} \mathcal{P}(N) N^{2}\left[Y_{N}^{2}-1\right] \\
& \cong 1+\frac{4 \theta}{\langle N\rangle}\left[\left[\left\langle N^{2}\right\rangle-\frac{\left\langle N^{3}\right\rangle}{\langle N\rangle}\right]=1-4 \theta .\right.
\end{aligned}
$$


With $\theta=0.04$ (70) one obtains from Eqs. (75) and (76):

$$
\Sigma[P, N] \cong 1.0032, \quad \Delta[P, N] \cong 0.8400
$$

The results of our approximate analytical calculations (77) may be compared with the full MC calculations (56).

Note that the correlation 69 between $T_{N}$ and $N$ leads to the additional term to $\Sigma(75)$ proportional to $\theta^{2}$, whereas $\Delta(76)$ includes a linear $\theta$-term. Therefore, the $\Delta[P, N]$ measure is much more sensitive to the correlations (69) between $T_{N}$ and $N$ than $\Sigma[P, N]$ : the linear $\theta$ contribution is essentially larger than $\theta^{2}$ one, as $\theta \ll 1$. Besides, it is sensitive to a sign of $\theta$. Therefore, both suppression (at $\theta>0$ ) and enhancement (at $\theta<0$ ) effects for $\Delta[P, N]$ may be observed.

\section{MODEL EXAMPLES}

\section{A. Quantum Gases}

The strongly intensive fluctuation measures $\Delta\left[P_{T}, N\right]$ and $\Sigma\left[P_{T}, N\right]$ have been recently studied in Ref. 34 for the ideal Bose and Fermi gases within the grand canonical ensemble. As it was already noted in Ref. [33], the Boltzmann approximation satisfies the conditions of the IPM, i.e. Eq. (14) is valid. Quantum statistics introduces particle correlations and the following general relations have been found [34]:

$$
\begin{aligned}
& \Delta^{\text {Bose }}\left[P_{T}, N\right]<\Delta^{\text {Boltz }}=1<\Delta^{\text {Fermi }}\left[P_{T}, N\right], \\
& \Sigma^{\text {Fermi }}\left[P_{T}, N\right]<\Sigma^{\text {Boltz }}=1<\Sigma^{\text {Bose }}\left[P_{T}, N\right]
\end{aligned}
$$

i.e. Bose statistics makes $\Delta\left[P_{T}, N\right]$ to be smaller and $\Sigma\left[P_{T}, N\right]$ larger than unity, whereas Fermi statistics works in exactly opposite way. The Bose statistics of the pion gas appears to be the main source of quantum statistics effects in the hadron gas with the temperature typical for the hadron system created in $\mathrm{A}+\mathrm{A}$ collisions. It gives approximately $\Delta\left[P_{T}, N\right] \cong 0.8$ and $\Sigma\left[P_{T}, N\right] \cong 1.1$, at $T \cong 150 \mathrm{MeV}$, i.e. suppression of $\Delta\left[P_{T}, N\right]$ and enhancement of $\Sigma\left[P_{T}, N\right]$ in a comparison to the Boltzmann approximation, equal to the IPM results (14). 
Fermi statistics contributions to $\Delta\left[P_{T}, N\right]$ and $\Sigma\left[P_{T}, N\right]$ for the protons are almost negligible for typical temperatures and baryonic chemical potentials in the hadron gas created in $\mathrm{A}+\mathrm{A}$ collisions.

\section{B. UrQMD}

In this subsection we discuss the UrQMD [39] results. In Ref. [33] the simulations for $\Delta\left[P_{T}, N_{-}\right]$and $\Sigma\left[P_{T}, N_{-}\right]$, where $N_{-}$is the number of negative particles, were considered. In the sample of $7 \%$ most central Xe+La collisions the fluctuation measure $\Sigma\left[P_{T}, N_{-}\right]$appears to be close to 1 for the whole SPS energy region $E_{l a b}$ from 20 to $158 \mathrm{GeV}$ per nucleon, whereas the fluctuation measure $\Delta\left[P_{T}, N_{-}\right]$increases with the collision energy from the value of 1 at $E_{l a b}=20 \mathrm{GeV}$ per nucleon to approximately 1.4 at $E_{l a b}=158 \mathrm{GeV}$ per nucleon. Note that the UrQMD takes into account several sources of fluctuations and correlations, e.g., exact conservation laws, resonance decays, flow effects, etc.

We use the UrQMD simulations in $\mathrm{Pb}+\mathrm{Pb}$ collisions at $E_{l a b}=20 \mathrm{GeV}$ per nucleon to study $\Delta\left[P_{T}, N_{-}\right]$and $\Sigma\left[P_{T}, N_{-}\right]$. With this example we illustrate effects of the centrality selection and limited detector acceptance and efficiency in $\mathrm{A}+\mathrm{A}$ collisions. The results presented in Fig. 5 correspond to the centrality bins of $5 \%, 10 \%, 15 \%$, and $20 \%$ most central $\mathrm{Pb}+\mathrm{Pb}$ collisions. One observes very strong increase of $\omega\left[N_{-}\right]$with a width of the centrality bin. This reflects the fact that fluctuations of the number of nucleon participants affect strongly the fluctuations of final hadron multiplicities. Therefore, scaled variances as the fluctuation measures become almost useless for wide centrality bins. For these wide samples of collisions, the scaled variances do not describe physical properties of the system but reflect the system size fluctuations (see more details in Ref. [8]). The strongly intensive measures $\Delta\left[P_{T}, N_{-}\right]$and $\Sigma\left[P_{T}, N_{-}\right]$look much more appropriate. These quantities are not sensitive to the trivial system size fluctuations. Their dependence on the size of the centrality bin is rather moderate (it is more pronounced for $\left.\Delta\left[P_{T}, N_{-}\right]\right)$and reflects changes in local physical properties for different centrality samples.

Another important aspect of today measurements of the e-by-e fluctuations in $\mathrm{A}+\mathrm{A}$ collisions is a limited detector acceptance and/or efficiency. Particles may be lost due to the geometry of the detector (for example fixed target experiments typically cover only forward hemisphere) 

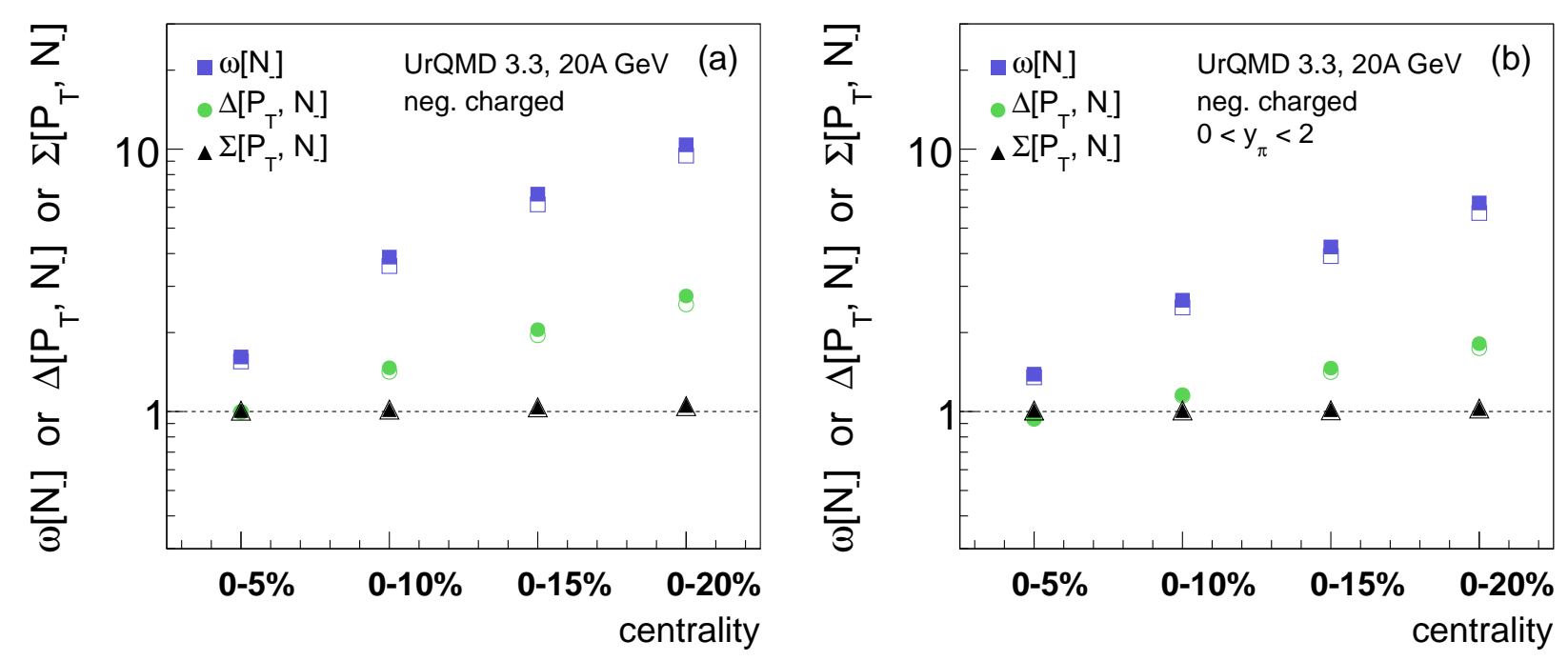

Figure 5: (Color online) The UrQMD results for the centrality dependence of $\omega\left[N_{-}\right]$(squares), $\Delta\left[P_{T}, N_{-}\right]($circles $)$, and $\Sigma\left[P_{T}, N_{-}\right]$(triangles) in $\mathrm{Pb}+\mathrm{Pb}$ collisions at $E_{l a b}=20 \mathrm{~A} \mathrm{GeV}$. A centrality selection is done with a restriction on the impact parameter $b$. (a): The full $4 \pi$ detector acceptance. (b): Only particles with center of mass rapidity in the interval $1<y_{\pi}<2$ are accepted (pion mass was assumed for all particles). Open symbols correspond to the case when $10 \%$ of particles was randomly rejected.

and we call it acceptance losses. On the other hand, even in this accepted kinematic region we still may have efficiency losses due to track reconstruction problems (including problems with ionization energy loss, $d E / d x$, reconstruction).

The UrQMD results for negatively charged particles in $\mathrm{Pb}+\mathrm{Pb}$ collisions at $E_{l a b}=20 \mathrm{~A} \mathrm{GeV}$ for the full $4 \pi$ acceptance and for the particles accepted only in the center of mass rapidity interval $1<y_{\pi}<2$ are shown in Fig. 5 (a) and (b), respectively (full symbols). From a comparison of the results for the full and limited detector acceptance one observes rather strong effects of acceptance losses for the scaled variance $\omega\left[N_{-}\right]$. The strongly intensive measures $\Delta\left[P_{T}, N_{-}\right]$and $\Sigma\left[P_{T}, N_{-}\right]$look again more appropriate. The effects of the limited acceptance are rather moderate for $\Delta\left[P_{T}, N_{-}\right]$and almost absent for $\Sigma\left[P_{T}, N_{-}\right]$. We also would like to stress that the acceptance dependence shown in Fig. 5 is the example only. In general, the measured 
magnitude of $\omega[N], \Delta\left[P_{T}, N\right]$ or $\Sigma\left[P_{T}, N\right]$ depends on both the correlation(s) length(s) and the size of the acceptance region (when the kinematic acceptance is much smaller that the correlation range the effect will be washed out). Therefore, when comparing experimental results to models the experimental kinematic restrictions should be carefully taken into account.

Finally, the example of the effect of efficiency losses is shown by open symbols in Fig. 5. In this case from each event we randomly rejected $10 \%$ of particles. As seen, the effect of efficiency losses is small or even negligible (comparison of full and open symbols) for all presented fluctuation measures but, in general, it depends on the fraction of rejected particles.

\section{SUMMARY}

In the present paper strongly intensive measures of the event-by-event fluctuations $\Delta\left[P_{T}, N\right]$ and $\Sigma\left[P_{T}, N\right]$ are studied. The recently proposed special normalization for these fluctuation measures are used, and it ensures that these measures are dimensionless and yields a common scale required for a quantitative comparison of fluctuations. Several phenomenological models are considered using the Monte Carlo simulations and analytical calculations. Our studies include different versions of the model of independent sources: with fixed number of sources, with the Poisson distribution of the number of sources, and with the Negative Binomial distribution. The quantities $\Delta\left[P_{T}, N\right]$ and $\Sigma\left[P_{T}, N\right]$ are found to be independent of the average number of sources and of its fluctuations. This reflects the strongly intensive properties of the $\Delta$ and $\Sigma$ measures, and is a main motivation of their using for the analysis of the event-by-event fluctuations in nucleus-nucleus collisions. The transverse momentum distribution of particles emitted from the source are assumed to be a thermal-like (Boltzmann) distribution over transverse mass. The average single-particle transverse momentum is then controlled by the inverse slope (temperature) parameter.

The system of sources with constant temperature appear to be equivalent to the model of independent sources, i.e. a relation $\Delta\left[P_{T}, N\right]=\Sigma\left[P_{T}, N\right]=1$ is obtained. For independent temperature fluctuations from source to source, one finds the correlations between transverse momenta of particles emitted from the same source. This leads to $\Delta\left[P_{T}, N\right]=\Sigma\left[P_{T}, N\right]=1+q_{S}$, where the value of $q_{S}$ is positive and depends only on the parameters of a single source. If all 
sources have the same fluctuating temperature, the model of independent sources becomes no more applicable. One obtains $\Delta\left[P_{T}, N\right]=\Sigma\left[P_{T}, N\right]=1+Q_{S}$, where the value of $Q_{S}$ increases linearly with the average number of sources $\left\langle N_{S}\right\rangle$ and increases with $\sigma_{T}$ which determines the size of temperature fluctuations.

A model which introduces a correlation between the temperature parameter and particle multiplicity is studied. In this case, the different values for the $\Delta$ and $\Sigma$ measures have been found: $\Delta\left[P_{T}, N\right]=1+q_{\delta}$ and $\Sigma\left[P_{T}, N\right]=1+q_{\sigma}$. Analytical calculations under several

simplifying assumptions give: $q_{\delta} \cong-4 \theta$ and $q_{\sigma} \cong 2 \theta^{2}$, where the parameter $\theta$ describes the correlations between $T_{N}$ and $N$ according to $(69)$ and is assumed to be small, $|\theta| \ll 1$.

The UrQMD simulations for $\mathrm{Pb}+\mathrm{Pb}$ collisions at the collision energy $E_{l a b}=20 \mathrm{GeV}$ per nucleon are done and analyzed. With this example we illustrate a role of the centrality selection and limited detector acceptance and efficiency in $\mathrm{A}+\mathrm{A}$ collisions. We find that the strongly intensive quantities $\Delta\left[P_{T}, N\right]$ and $\Sigma\left[P_{T}, N\right]$ have an advantage over the standard fluctuation measures. In contrast to the scaled variance, $\Delta\left[P_{T}, N\right]$ and $\Sigma\left[P_{T}, N\right]$ demonstrate much weaker sensitivity to the width of the centrality bin and to the limited detector acceptance and efficiency.

In all considered model examples, $\Delta\left[P_{T}, N\right]$ appears to be more sensitive to interparticle correlations than $\Sigma\left[P_{T}, N\right]$. This reveals itself as stronger deviations of $\Delta\left[P_{T}, N\right]$ from the IPM results (14). Even for $\Delta\left[P_{T}, N\right]=\Sigma\left[P_{T}, N\right]$, in the MC simulations in Sec. III, a stronger sensitivity of $\Delta\left[P_{T}, N\right]$ manifests as its larger statistical errors.

We hope that the results obtained in this paper will be helpful to elucidate the properties of $\Delta\left[P_{T}, N\right]$ and $\Sigma\left[P_{T}, N\right]$ measures.

\section{Acknowledgments}

We would like to thank Marek Gaździcki, Laszlo Jenkovszky, and Stanislaw Mrówczyński for fruitful discussions and comments. We are indebted to the authors of the UrQMD model for the possibility to use their code in our analysis. The work of M.I.G. is supported by the State Agency of Science, Innovations and Informatization of Ukraine, contract F58/384-2013. The work of K.G. was supported by the the National Science Center, Poland grant DEC- 
2011/03/B/ST2/02617 and grant 2012/04/M/ST2/00816.

[1] M. Stephanov, K. Rajagopal, and E. V. Shuryak, Phys. Rev. D 60, 114028 (1999).

[2] F. Becattini, J. Manninen, and M. Gaździcki, Phys. Rev. C 73, 044905 (2006).

[3] M. Gaździcki [NA61/SHINE Collaboration], J. Phys. G 36, 064039 (2009).

[4] G. Odyniec [STAR Collaboration], J. Phys. G 35, 104164 (2008);

A. Adare et al. [PHENIX Collaboration], Phys. Rev. C 78, 044902 (2008).

[5] V. P. Konchakovski, M. I. Gorenstein, E. L. Bratkovskaya, and W. Greiner, J. Phys. G 37, 073101 (2010).

[6] M. I. Gorenstein and M. Gaździcki, Phys. Rev. C 84, 014904 (2011).

[7] K. Grebieszkow, Acta Phys. Polon. B 43, 1333 (2012).

[8] V. P. Konchakovski, V. V. Begun, M. I. Gorenstein, and E. L. Bratkovskaya, J. Phys. G 40, 045109 (2013)

[9] M. Gaździcki and S. Mrówczyński, Z. Phys. C 54, 127 (1992).

[10] H. Appelshauser et al. [NA49 Collaboration], Phys. Lett. B 459, 679 (1999).

[11] T. Anticic et al. [NA49 Collaboration], Phys. Rev. C 70, 034902 (2004).

[12] C. Alt et al. [NA49 Collaboration], Phys. Rev. C 70, 064903 (2004).

[13] T. Anticic et al. [NA49 Collaboration], Phys. Rev. C 79, 044904 (2009).

[14] D. Adamova et al. [CERES Collaboration], Nucl. Phys. A 727, 97 (2003).

[15] K. Adcox et al. [PHENIX Collaboration], Phys. Rev. C 66, 024901 (2002).

[16] M. R. Atayan et al. [EHS/NA22 Collaboration], Phys. Rev. Lett. 89, 121802 (2002).

[17] M. Bleicher, M. Belkacem, C. Ernst, H. Weber, L. Gerland, C. Spieles, S. A. Bass and H. Stoecker et al., Phys. Lett. B 435, 9 (1998).

[18] S. Mrówczyński, Phys. Lett. B 439, 6 (1998).

[19] F. Liu, A. Tai, M. Gazdzicki and R. Stock, Eur. Phys. J. C 8, 649 (1999).

[20] S. Mrówczyński, Phys. Lett. B 459, 13 (1999).

[21] S. Mrówczyński, Phys. Lett. B 465, 8 (1999).

[22] S. Mrówczyński, Acta Phys. Polon. B 31, 2065 (2000). 
[23] O. V. Utyuzh, G. Wilk and Z. Wlodarczyk, Phys. Rev. C 64, 027901 (2001).

[24] R. Korus and S. Mrówczyński, Phys. Rev. C 64, 054906 (2001).

[25] R. Korus, S. Mrówczyński, M. Rybczynski and Z. Wlodarczyk, Phys. Rev. C 64, 054908 (2001).

[26] J. Zaranek, Phys. Rev. C 66, 024905 (2002).

[27] O. Pruneau, S. Gavin and S. Voloshin, Phys. Rev. C 66, 044904 (2002).

[28] S. Mrówczyński and E. V. Shuryak, Acta Phys. Polon. B 34, 4241 (2003).

[29] S. Mrówczyński, M. Rybczynski, and Z. Włodarczyk, Phys. Rev. C 70, 054906 (2004).

[30] Q. H. Zhang, L. Huo, W. N. Zhang, L. Huo and W. N. Zhang, Phys. Rev. C 72, 047901 (2005).

[31] K. Grebieszkow, Phys. Rev. C 76, 064908 (2007).

[32] W. M. Alberico and A. Lavagno, Eur. Phys. J. A 40, 313 (2009).

[33] M. Gaździcki, M. I. Gorenstein, and M. Mackowiak-Pawlowska, Phys. Rev. C 88, 024907 (2013).

[34] M. I. Gorenstein and M. Rybczynski, arXiv:1308.0752 [nucl-th], Phys. Lett. B, in print.

[35] T. Czopowicz [NA61 Collaboration], "Results from NA61/SHINE ion program", slides from 16th Lomonosov Conference on Elementary Particle Physics, Moscow, August 2013; http://www.icas.ru/english/LomCon/16lomcon/16lomcon_programme.htm;

P. Seyboth [NA49 and NA61 Collaborations], "Recent results from the search for the critical point of strongly interacting matter at the CERN SPS", slides from XLIII International Symposium on Multiparticle Dynamics (ISMD), Chicago, September 2013, https://indico.hep.anl.gov/indico/conferenceDisplay.py?confId $=0$.

[36] A. Bialas, M. Bleszynski, and W. Czyz, Nucl. Phys. B 111, 461 (1976).

[37] V. V. Begun, M. Gazdzicki, and M. I. Gorenstein, Phys. Rev. C 78, 024904 (2008).

[38] G. Wilk and Z. Włodarczyk, Phys. Rev. C 79, 054903 (2009); J. Phys. G 38, 065101 (2011); Eur. Phys. J. A 48, 161 (2012).

[39] S. A. Bass et al., Prog. Part. Nucl. Phys. 41, 255 (1998);

M. Bleicher et al., J. Phys. G 25, 1859 (1999). 